\title{
Research Paper \\ Effects of Early Morning Exercise on Serum Brain-Derived Neurotrophic Factor Level and Its Relation With Blood Cholesterol and Glucose Levels in the Elderly Men
}

*Vahid Valipour Dehnou1${ }^{1}$, Mehdi Abbasi Moghadamㄹ, Mohammad Soleymani Farsani ${ }^{1}$

1. Department of Sports Sciences, Faculty of Literature and Humanities, Lorestan University, Khorramabad, Iran.

\begin{tabular}{|l|l|l|l}
\hline $\begin{array}{c}\text { Use your device to scan } \\
\text { and read the article online }\end{array}$ & $\begin{array}{l}\text { Citation: Valipour Dehnou V, Abbasi Moghadam M, Soleymani Farsani M. [Effects of Early Morning Exercise on Serum } \\
\text { Brain-Derived Neurotrophic Factor Level and Its Relation With Blood Cholesterol and Glucose Levels in the Elderly Men (Per- } \\
\text { sian)]. Iranian Journal of Ageing. 2018; 13(3):324-333. https://doi.org/10.32598/SIJA.13.3.324 }\end{array}$ \\
doi: : https://doi.org/10.32598/SIJA.13.3.324
\end{tabular}

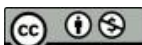

Funding: See Page 332

Received: 4 Apr 2018

Accepted: $28 \mathrm{Jul} 2018$

Keywords

Morning exercise,

Elderly men, Cho-

lesterol, Glucose,

BDNF

\section{ABSTRACT}

Objectives Early morning exercise is prevalent in Iranian population, and yet no study has assessed its effect on brain-derived neurotrophic factor. Therefore, the purpose of this study was to assess the effect of early morning exercise on serum levels of brain-derived neurotrophic factor and its relation with cholesterol levels in the elderly males in Khorramabad City, Iran.

Methods \& Materials In this quasi-experimental study, 13 older men (mean [SD] age: 56.46[3.93] y; mean [SD] weight 82.46[8.23] kg; mean [SD] height: $175.46[5.17] \mathrm{cm}$ ) voluntarily participated. In the training day, at first, blood samples were obtained at 07:30 AM in fasting state. Then, subjects performed training protocol for 50 min duration that included 15 min warm-up, 25 min main activity, and 10 min cool-down, and after three min, subsequent blood samples were obtained. To measure serum levels of glucose, cholesterol, the Brain-Derived Neutrophilic Factor (BDNF) enzymatic and ELISA methods were used, respectively. To analyze the obtained data, paired-samples $t$ test and Pearson correlation were used, and statistical significance was set at $\mathrm{P}<0.05$.

Results Results showed that serum concentration of BDNF ( $P=0.002)$ and glucose $(P=0.01)$ increased significantly. Also, there were no significant correlations between serum concentration of BDNF and glucose in both pretest and posttest $(P>0.05)$. However, there were near significant negative correlation between serum concentration of BDNF and cholesterol in pretest $(r=-0.53, P=0.061)$ and significant negative correlation in posttest ( $r=-0.703, \mathrm{P}=0.007)$.

Conclusion Early morning exercise for approximately 50 minutes increases serum concentration of BDNF and glucose in elderly males. Also, there is no significant positive correlation between them. But, there is significant negative correlation between serum concentration of BDNF and cholesterol. Therefore, early morning exercise for the elderly men is recommended to improve brain health. Also, older men, if they have normal cholesterol levels, will get more benefits from early morning exercise.

\section{* Corresponding Author:}

Vahid Valipour Dehnou, PhD

Address: Department of Sports Sciences, Faculty of Literature and Humanities, Lorestan University, Khorramabad, Iran.

Tel: +98 (916) 6691874

E-mail: valipour.v@lu.ac.ir 


\section{Extended Abstract}

\section{Objectives}

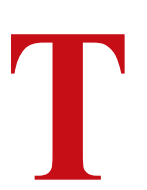

he relationship between regular participation in physical activity, especially aerobic exercise, and a variety of cognitive benefits have been already suggested [1-3]. Precise neurobiological mechanisms are unclear in improvement of cognitive functions, but studies on rodents have suggested the role of specific molecules such as Brain-Derived Neurotrophic Factor (BDNF), Insulinlike Growth Factor 1 (IGF-1) and Vascular Endothelial Growth Factor (VEGF) [1, 2].

In the brain, high-cholesterol diet reduces BDNF and neurodegeneration, and cause changes in long-term potentiation which lead to memory malfunction [4]. Studies have shown that plasma levels of BDNF and or its receptors reduce in older people and there is a negative relationship between BDNF levels and age [5]. The present study aimed to investigate the role of morning exercise on BDNF levels and its relationship with blood cholesterol and glucose levels.

\section{Methods and Materials}

This quasi-experimental study was conducted in Khorramabad City, Iran with a pretest and posttest design in 2017. Study population consists of 60 elderly men. Of this, 13 (mean [SD] age=56.46[3.93] year, mean $[\mathrm{SD}]$ weight $=82.46[8.23] \mathrm{kg}$, mean $[\mathrm{SD}]$ height $=175.46[5.17] \mathrm{cm}$ ) voluntarily participated in the study. Inclusion criteria were as follows: age over 50 , with physical and mental health, willingness to participate in the study, and no history of fracture in the last few months. Exclusion criteria were any signs of cardiovascular or physical problems during exercise and unwillingness to continue exercise.

The study procedure has been approved by the Ethics Committee of Lorestan University. The subjects were first warmed up for 15 minutes, including two rounds of walking, running slowly around the track, and then stretching movements. The main part of the exercise included 25 minutes of running at fast, moderate, and slow paces. To cool down, the subjects performed stretching movements for 10 minutes. The training sessions lasted 50 minutes. This protocol was the physical exercise program that the subjects performed earlier (for about 6 months, two sessions per week).

On the day of the training, the blood samples were first taken during a fasting state and then the exercise protocol was performed. Three minutes after the training session, the next blood samples were taken. The blood sample was centrifuged at $3500 \mathrm{rpm}$ for $5 \mathrm{~min}$ and the obtained serum was stored for later tests at $-30^{\circ} \mathrm{C}$. Serum BDNF concentrations were determined by ELISA assay using the appropriate kit (sensitivity $=0.063 \mathrm{ng} / \mathrm{mL}$, detection range $=0.325-20 \mathrm{ng} / \mathrm{mL}$, Casabau, Japan) according to the manufacturer's instructions. The levels of glucose and serum cholesterol were determined using a Adjust Diagnostics Kit (sensitivity= $1 \mathrm{mg} / \mathrm{dL}$, Ireland) in the Hitachi 917 automated analyzer. The obtained data were analyzed by paired $t$ test and Pearson correlation test.

\section{Result}

The results of serum BDNF, glucose and cholesterol levels of subjects and the results of paired $t$ test are presented in Table 1. These results indicate that serum BDNF $(\mathrm{P}=0.002)$ and glucose levels $(\mathrm{P}=0.01)$ significantly increased. There was no significant correlation between serum BDNF level and glucose level in all pretest and posttest conditions $(\mathrm{P}>0.05)$. Also, the difference between glucose and serum BDNF levels was not significant before and after the intervention $(\mathrm{P}=0.887, \mathrm{r}=-0.044)$, but there was a negative correlation between cholesterol level and serum BDNF levels before the intervention ( $\mathrm{r}=-0.53$, $\mathrm{P}=0.061)$, and after the intervention $(\mathrm{P}=0.007, \mathrm{r}=-0.703)$. The beneficial effects of physical activity can be partly explained by inducing BDNF gene expression in the hippocampus $[4,6,7]$.

Also, these effects can be different depending on the type of exercise and some factors, including blood cholesterol and glucose levels $[2,4]$. The results of eating

Table 1. The $t$ test results of study participants

\begin{tabular}{clcccccccc}
\hline & \multicolumn{1}{c}{ BDNF (ng.mL) } & & & & Glucose (mg.dL) & & Cholesterol (mg.dL) \\
\hline Pretest & Posttest & t & P & Pretest & Posttest & t & P & Pretest \\
\hline $13.1 \pm 54.07$ & $14.0 \pm 46.61$ & -3.052 & 0.002 & $79.7 \pm 23.90$ & $87.15 \pm 46.39$ & -3.874 & 0.01 & $176.31 \pm 62.59$
\end{tabular}


breakfast before morning exercise are controversial. Although in competitive athletes, carbohydrate ingestion is suggested 2-4 hours before the exercise in the morning, but in recreational athletes that exercise with moderate intensity, it is not very common. In addition, many people refuse to eat breakfast before exercise due to increase in fat metabolism [8].

Although the fat oxidation rate was not calculated in this study, the main aim of this study was to evaluate the effect of exercise in the early morning on serum BDNF level as a measure of cognitive performance whose results showed that moderate-intensity exercise early in the morning could increase serum BDNF levels and could be useful for mental health, and hence, improving cognitive functions in active elderly men. Food deprivation/fasting can also induce expression of BDNF level in neural circuits involved in recognition by increasing their activity and switching cellular energy substrate utilization from glucose to ketones [7].

From an evolutionary point of view, this activitydependent production of BDNF likely contributes to optimization of brain function during fasting and running [7]. Therefore, BDNF production during fasting may be a mechanism for controlling energy substrate. considering the role of physical activity and fasting in increasing the expression of BDNF, the exercise in the early morning can be helpful in term of producing more BDNF.

\section{Conclusion}

One of the goals of exercising early in the morning is to burn more fat, because studies have shown that early exercise increases the oxidation of fat more than evening exercise [9]. Part of fat oxidation is due to increased BDNF secretion, because the free part of BDNF is used for fat oxidation in the skeletal muscle and other part is entered into the brain through a bloodbrain barrier [5], and can have beneficial effects on brain structure and function. The results of this study showed that exercise in fasting state can increase BDNF levels in active elderly men. This increase in BDNF levels can be affected by cholesterol levels. Therefore, lowering cholesterol levels through exercise or by reducing the consumption of high-cholesterol foods can be important in terms of brain and mental health in physically active elderly men.

\section{Ethical Considerations}

\section{Compliance with ethical guidelines}

The Ethics Committee of Lorestan University confirmed this research.

\section{Funding}

This research was extracted from a research project done in Lorestan University and was financially supported by Lores$\tan$ University.

\section{Conflict of interest}

The authors declared no conflict of interest.

\section{Acknowledgements}

Hereby, we appreciate all those who participated in this research. 


\title{
تأثير ورزش صبحكَاهى بر سطوح سرمى عامل رشد عصبى مشتق از مغز و ارتباط آن با سطوح كلسترول در مردان سالمند صبح
}

\author{
"وحيد ولىيور دهنو' 'ه، مهدى عباسى مقدم'، محمد سليمانى فارسانى' \\ 1 - كروه علوم ورزشى، دانشكده ادبيات و علوم انسانى، دانشكاه لرستان، خرم آباده ايران.
}

\begin{abstract}
حكيد

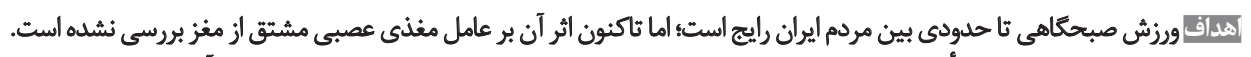

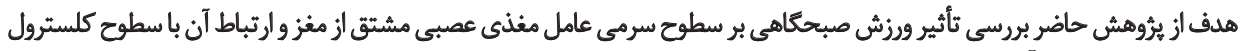

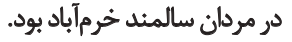

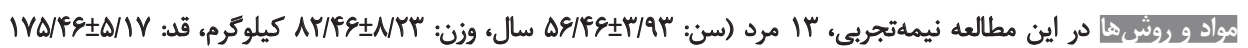

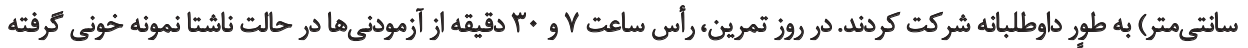

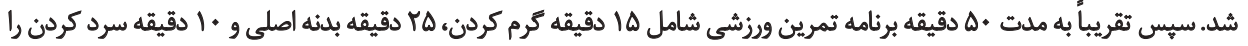

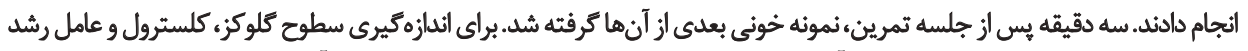

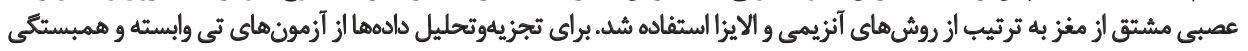

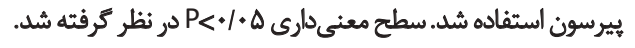

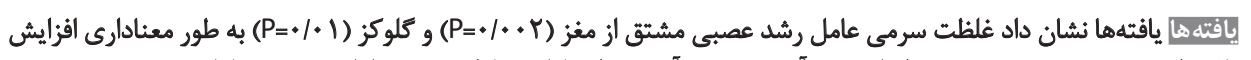

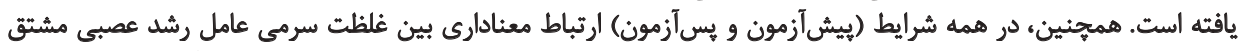

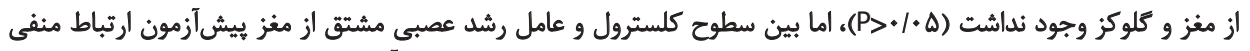

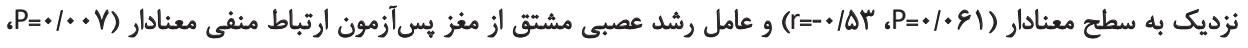

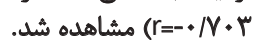

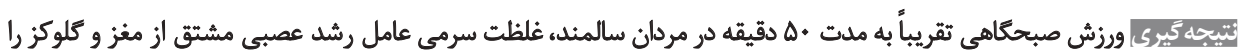

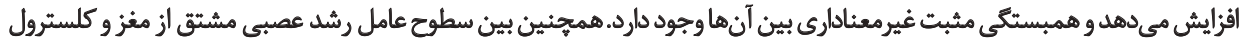

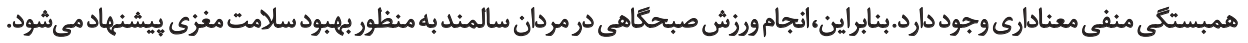

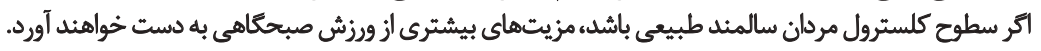

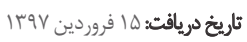

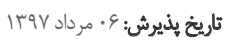

مشتق از مغز'، عامل رشد شبهانسولين-1' و و عامل رشد اندوتليال

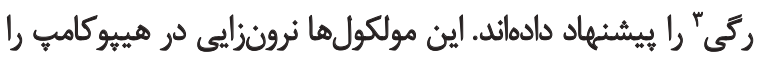

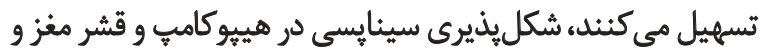

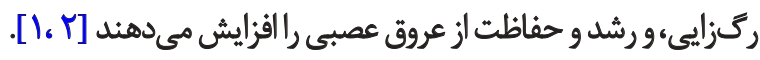

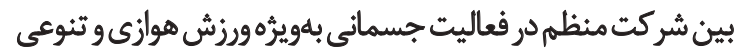

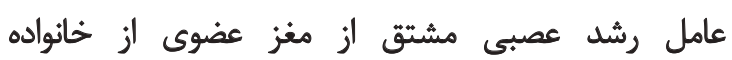

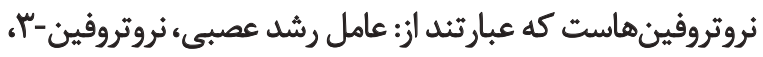

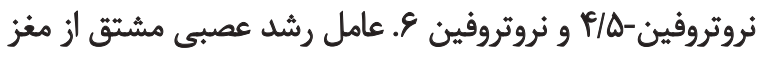

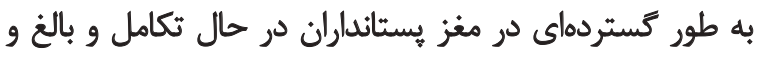

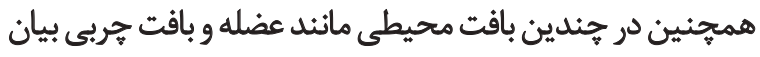

1. Brain-Derived Neurotrophic Factor (BDNF)

2. Insulin-like Growth Factor (IGF-1)

3. Vascular Endothelial Growth Factor (VEGF)

مقدمه

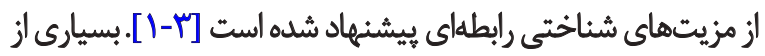

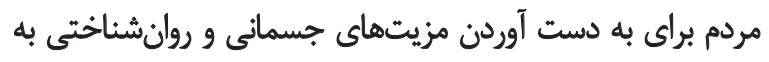

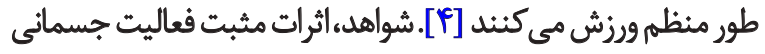

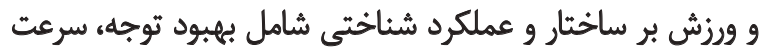

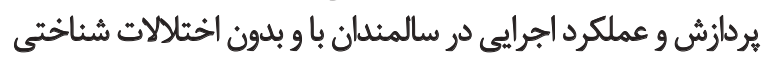

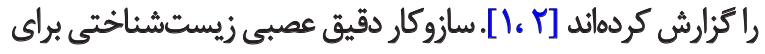

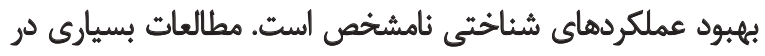

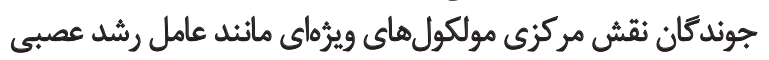

\section{.}

: نويسئده مسئول:

دكتر وحيد ولى ايليور دهنو

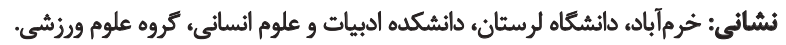
تلفن: 91 valipour.v@lu.ac.ir يست الكترونيكي 
وضعيتى ل) در سالمندان داراي اختلال ملايم شناختى نشان دادند

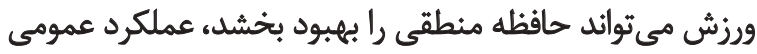

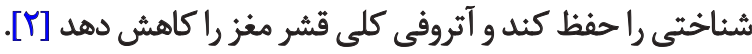

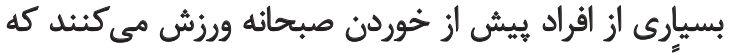

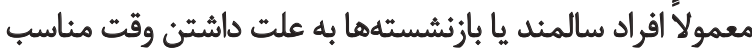

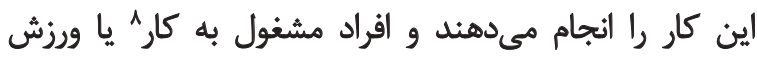

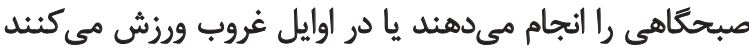

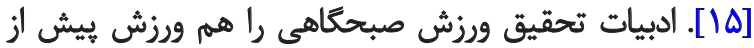

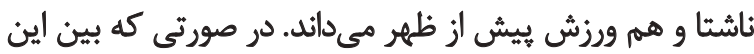

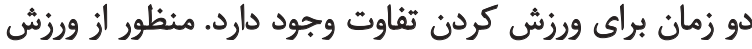

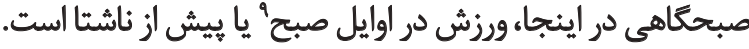

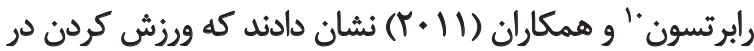

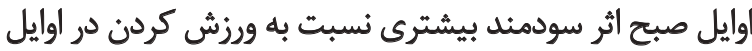

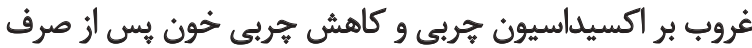

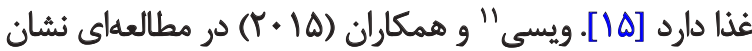

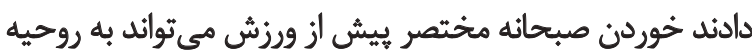
يس از ورزش و ميزان ذهنى اشتها در زنان فعال سود برساند [? أن]. سطوح كم كلسترول تام ييش از مداخله ورزشى با بهبود نمرات

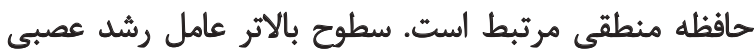

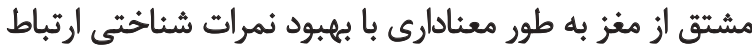

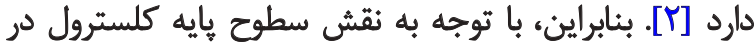

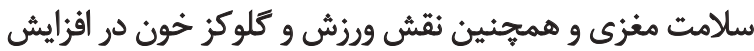

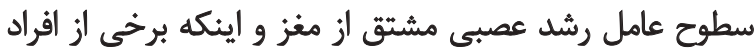

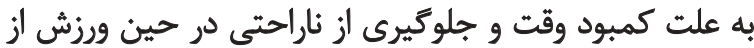

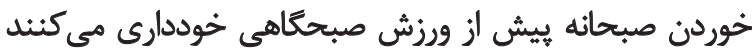

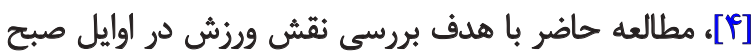

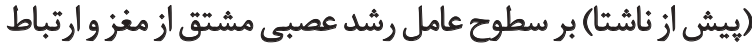

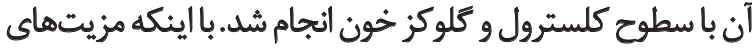

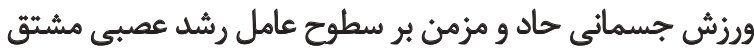

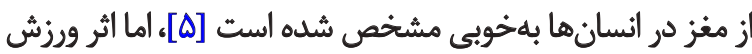

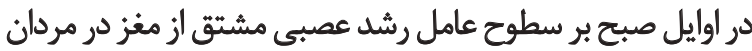

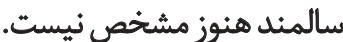

$$
\text { روش مطالعه }
$$

اين مطالعه نيمهتجربى با طرح ييشآزمون و يسآزمون در سال

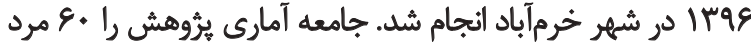

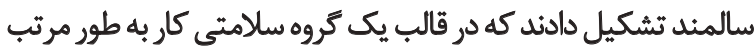

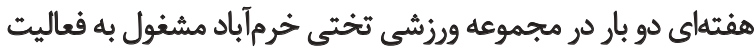

\section{Postural balance retraining}

8. Working population

9. Early morning exercise

10. Robertson

11. Veasey
مىشود [1]]. عامل رشد عصبى مشتق از مغز يك يروتئين مهم در

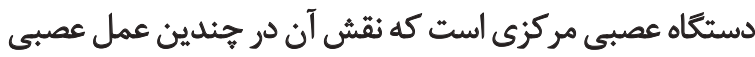

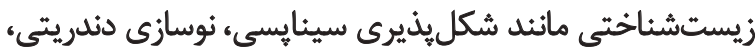

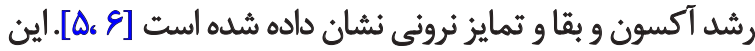

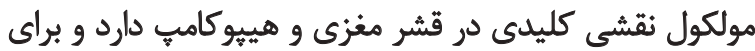

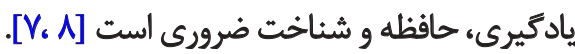
كلسترول مادهاى مومى و جربيىمانئد است كه در همانه

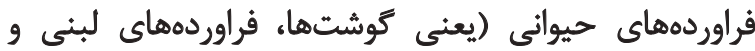

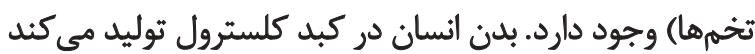

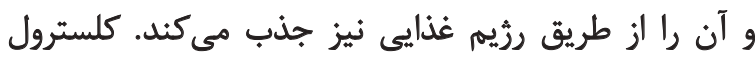

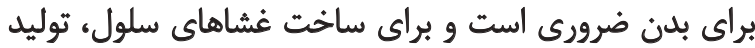

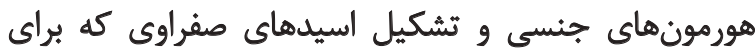

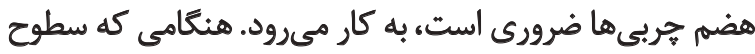

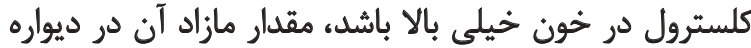

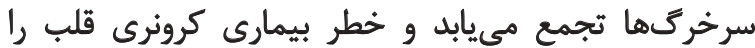

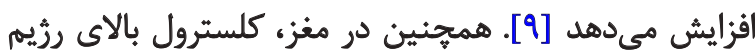

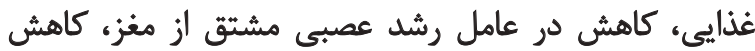

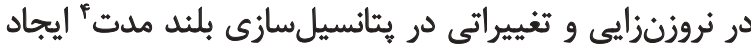

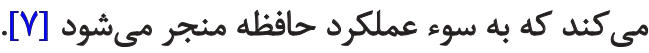
از طرفى سالمندى به عنوان كاهش طبيعى در عملكرد بافت،

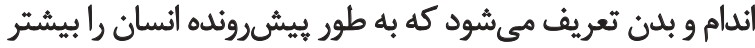

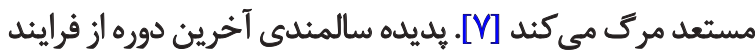

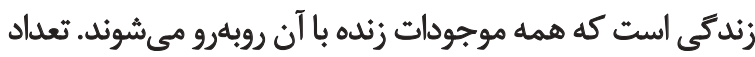

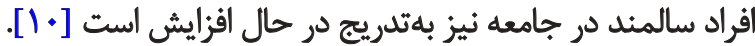

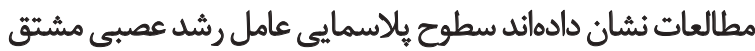

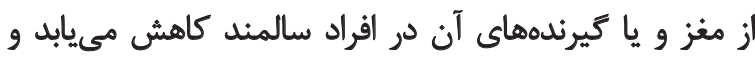

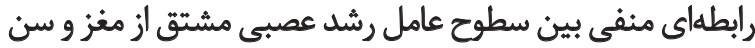

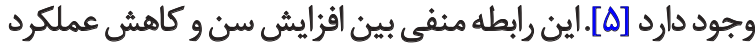

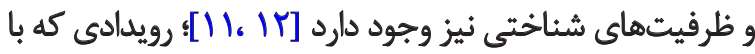

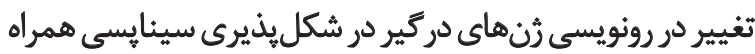

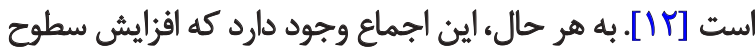

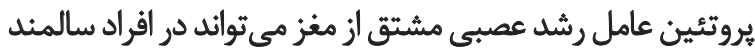

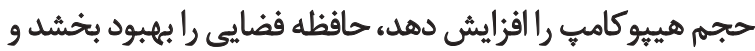

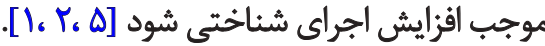

مطالعات نشان داداند ورزش هوازى [ه] و تمرين مقاومتى رئى

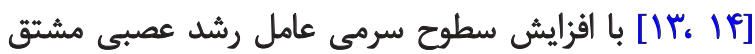

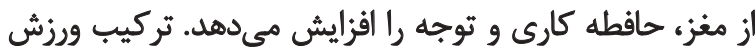

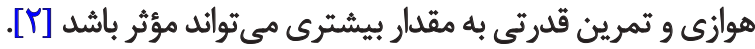

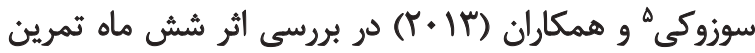

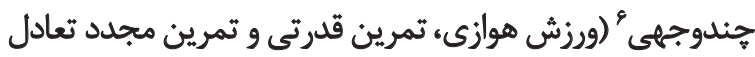

4. Long-term potentiation

5. Suzuki

6. Multicomponent exercise program 


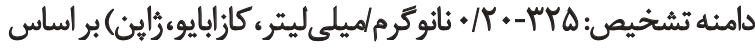

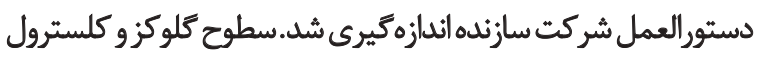

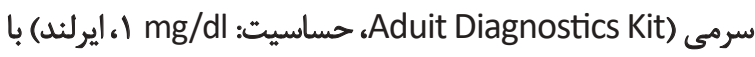
استفادها زروش آنزيمى بادستًاه هيتاجى آتوآنالايزر 91 تعيين شد.

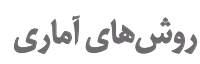

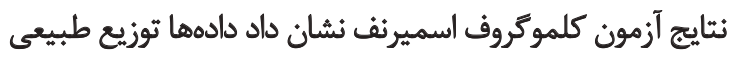

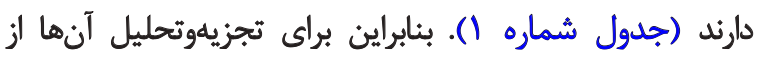

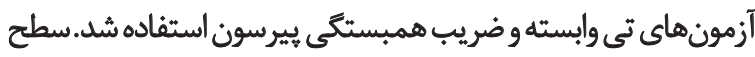
معنى دارى ه ٪ اء در نظر كرفته شد.

يافتهها

در اين مطالعه با مرد سالمند ساكن شهر خرمآباد بررسى شدئد.

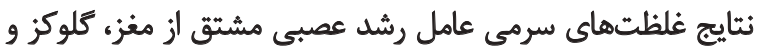

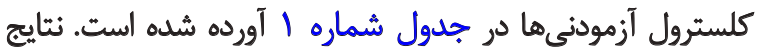

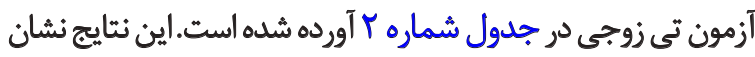

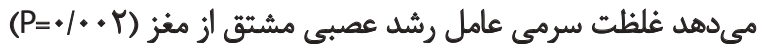
و كلوكز (1+1/

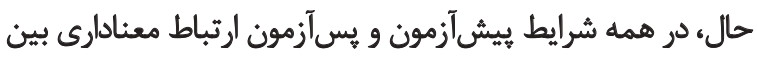

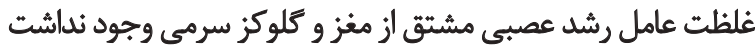

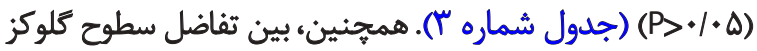

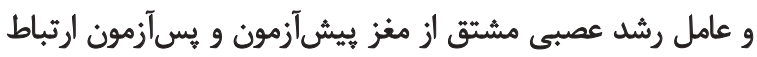

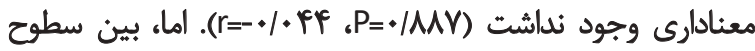

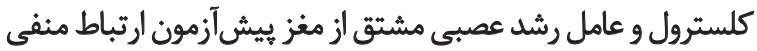

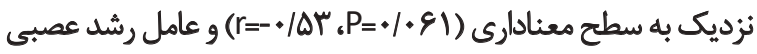

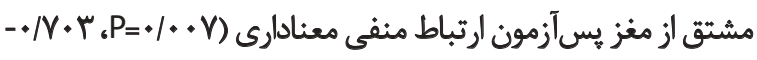
r=

بـ

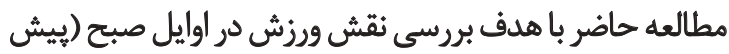

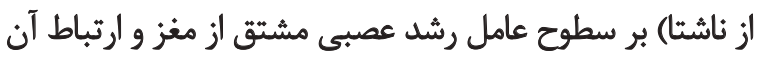

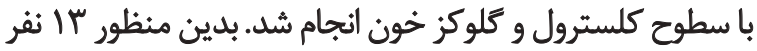

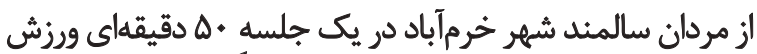

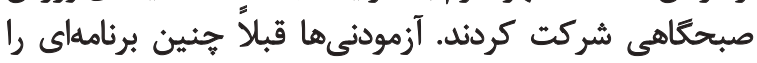

بودند. يَ از شرح كامل روش انجام ثُروهش و انجام نمونه كيرى

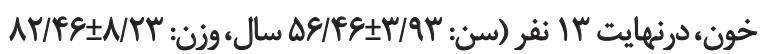
كيلوكرم، قد: IVA/IV

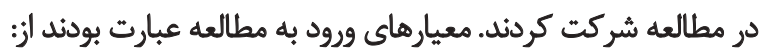

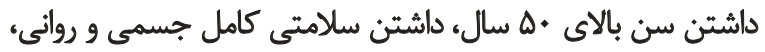

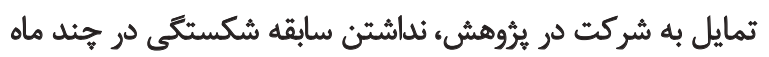

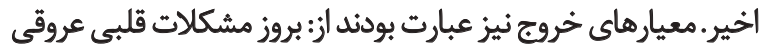

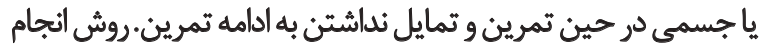

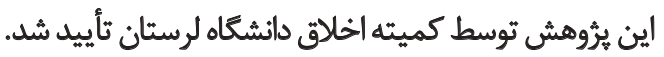

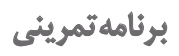

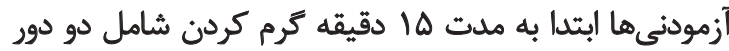

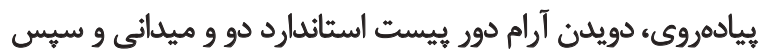

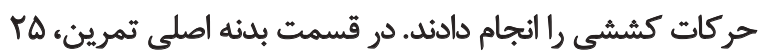

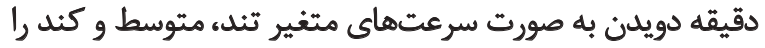

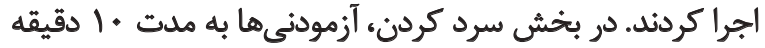

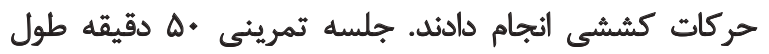

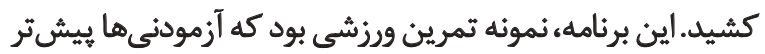

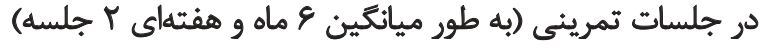

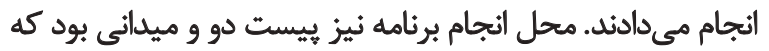

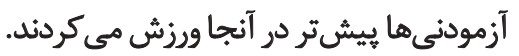

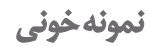

در روز تمرين ابتدا كارشناس آزمايشكاه از آزمودنىها در بر حالت

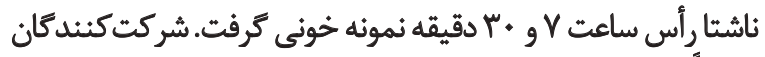

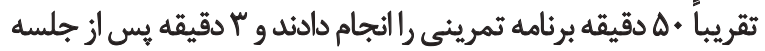

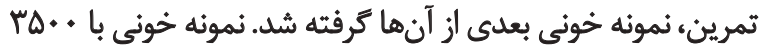

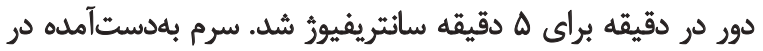

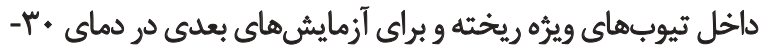
درجه سانتى كراد نتُهدارى شد.

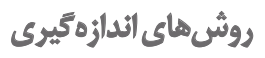

غلظتهاي سرمى عامل رشد عصبى مشتق از مغز بهروش الايزاو

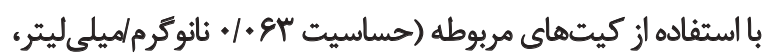

\begin{tabular}{|c|c|c|c|c|}
\hline \multicolumn{2}{|c|}{ 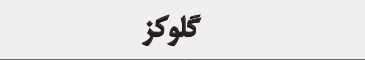 } & \multicolumn{2}{|c|}{ عامل رشد عصبى مشتق از مغز } & \multirow{2}{*}{ متغير } \\
\hline يس آزمون & ييش آزهون & 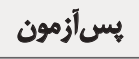 & ييش آزمون & \\
\hline.$/ T A$ &.$/ 1 \Delta$ &.$/ M r$ &.$/ M$ & آماره \\
\hline$\%$. &.$\pi$ &.$/ \pi$ &.$/ N$ & $P$ \\
\hline
\end{tabular}

جدول ا. نتايج آزمون كولموكروف اسميرنف

㕰 
جدول r. نتايج آمار توصيفى (ميانكين \انحراف معيار) و استنباطى

\begin{tabular}{|c|c|c|c|c|c|c|c|c|}
\hline \multirow{2}{*}{ كلسترول (mg/dl) } & \multicolumn{4}{|c|}{ كلوكز (mg/dl) } & \multicolumn{4}{|c|}{ عامل رشد عصبى مشتق از مغز (ng/ml) } \\
\hline & $\mathbf{P}$ & $\mathrm{t}$ & يس أزمون & بيش آزمون & $\mathbf{P}$ & $t$ & 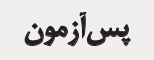 & يشي أزمون \\
\hline$|V E / H| \pm C r / \Delta q$ & $+1+1$ & $-r / A V^{e}$ & $A V / \backslash \Delta \pm E \& / T^{q}$ & $V q / V \pm r T / q$. & $\%$ & $-r / \circ \Delta r$ & $|f / + \pm x+8|$ & $\mid r / \backslash \pm \Delta F / \cdot V$ \\
\hline
\end{tabular}

少

شاخص توده بدنى، جربى شكمى، ترى گكليسريد، كلسترول تام

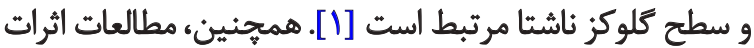

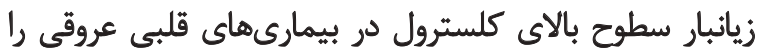

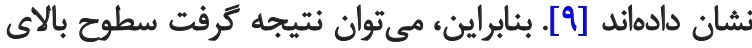

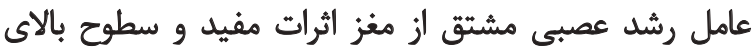
كلسترول اثرات مضرى در ايجاد بيمارى هاى قار ملبى عروقى دارند.

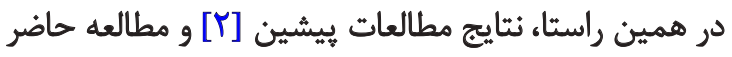

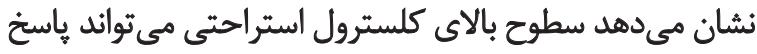

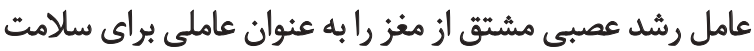

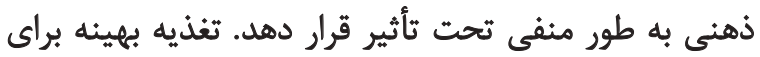

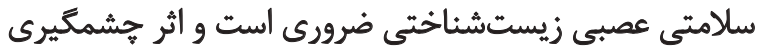

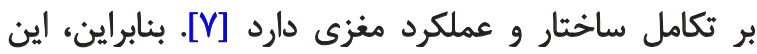

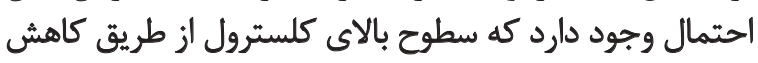

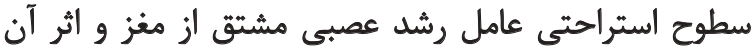

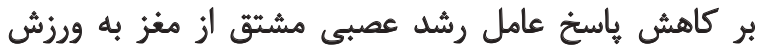

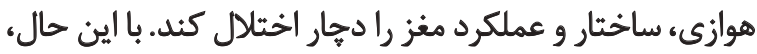

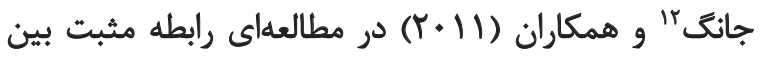

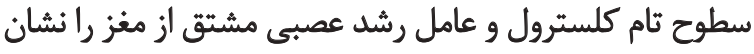

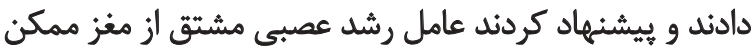

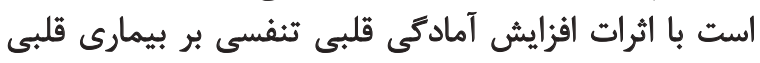

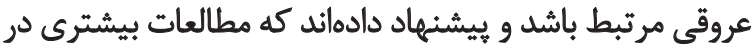

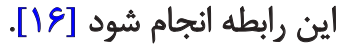

عامل رشد عصبى مشتق از مغز ممكن است سوختوساز

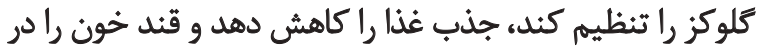

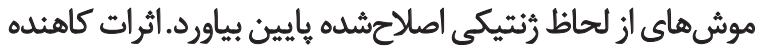

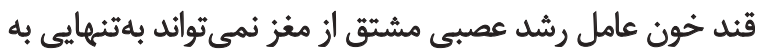

12. Jung
هفتهاى دو بار انجام مى دادند.نتايج مهم مطالعه حاضر عبارتند ازز:

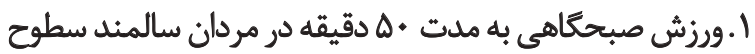

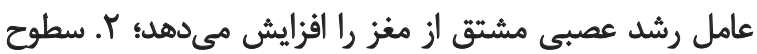

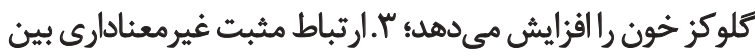

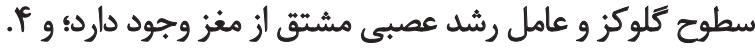

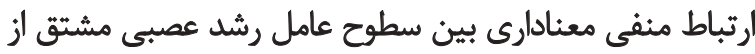
مغز و كلسترول سرمى استراحتى وجود دين دارد.

مطالعات نشان دادهاند ورزش مي تواند سلامت ذهنى و ومغزي

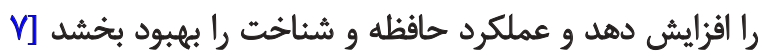
[F.

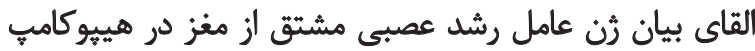

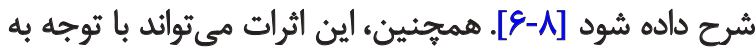

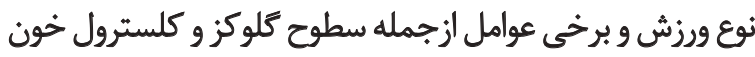

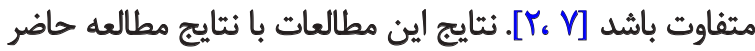
همسو است.

ورزش به طور حاد و مزمن بهويرهه با شدت متوسط و بالاتر

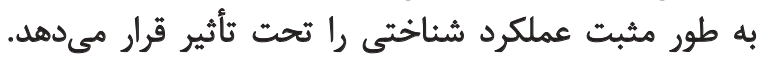

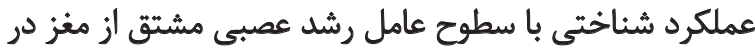

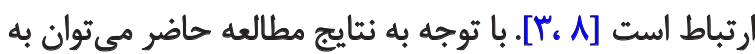

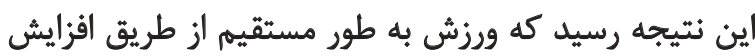

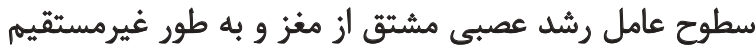

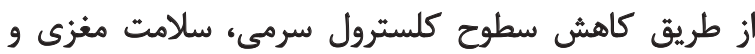

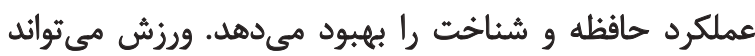

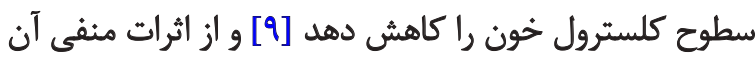

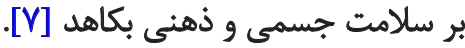

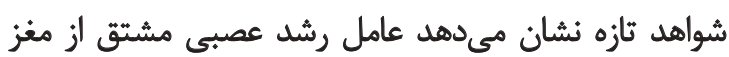

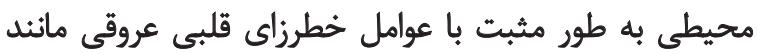

جدول با. نتايج آزمون ضريب همبستكى يمرسون

\begin{tabular}{|c|c|c|c|c|c|c|}
\hline \multirow{2}{*}{\multicolumn{2}{|c|}{ كلسترول }} & \multicolumn{4}{|c|}{ كملوكز } & \multirow{3}{*}{ 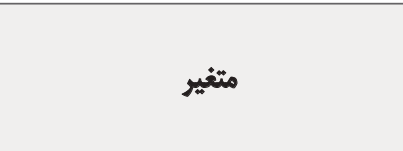 } \\
\hline & & \multicolumn{2}{|c|}{ يسآزمون } & \multicolumn{2}{|c|}{ يشيش آزمون } & \\
\hline $\mathbf{r}$ & $\mathbf{P}$ & $\mathbf{r}$ & $\mathbf{P}$ & $\mathbf{r}$ & $\mathbf{P}$ & \\
\hline.$- / \Delta M T$ & +1.91 & . Mar & ./rer & +Fro & $+/$ IFY & عامل رشد عصبى مشتق از مغز ييش آزمون \\
\hline$-\cdot / V \cdot r$ & $+1+\bullet V$ & +/Atr & .1810 & . IrTe & +/Far & عامل رشد عصبى مشتق از مغز يسآزمون \\
\hline
\end{tabular}

L 
نتايج در زمينه مصرف صبحانه مختصر بيش از ورزش

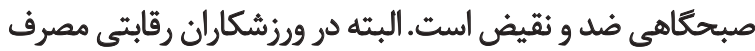

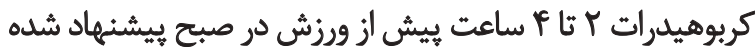

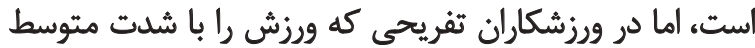

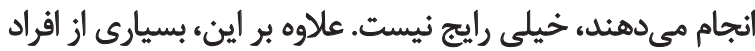

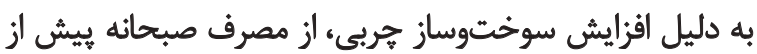

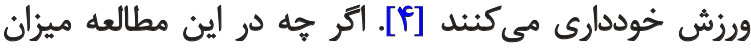
اكسيداسيون جربى محاسبه نشده است (جزء اهدئ اهداف مطالعه

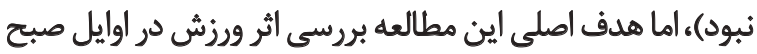

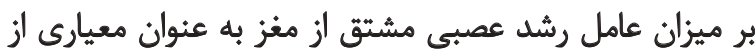

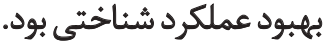

نتايج نشان داد ورزش با شدت متوسط در اوايل صبح مى تواند

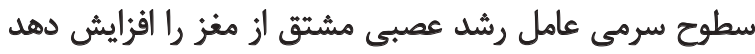

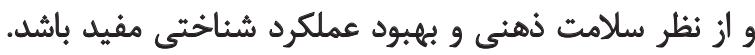

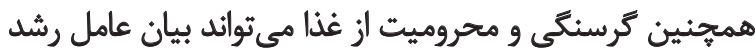

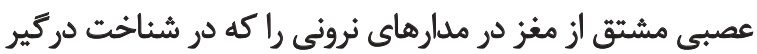

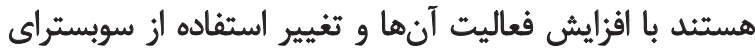

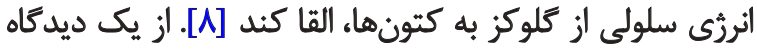

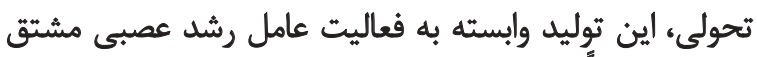

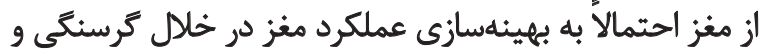

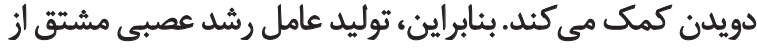

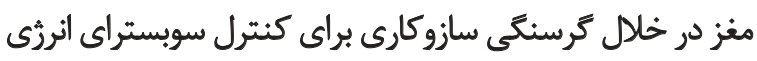

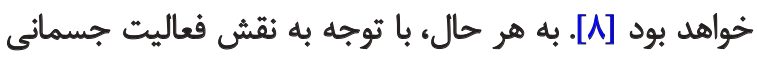

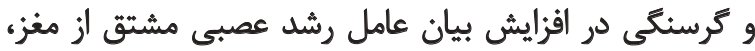

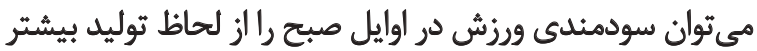
عامل رشد عصبى مشتق از مغز مفيد دانست دوريت

ويسى و همكاران (10 (T) نشان دادند از لحاظ ميزان احساس

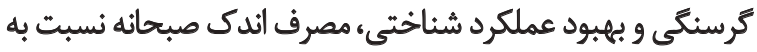

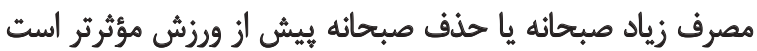

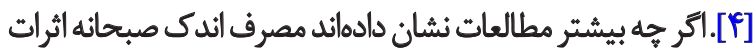

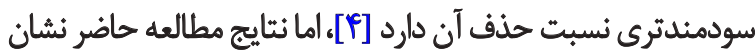

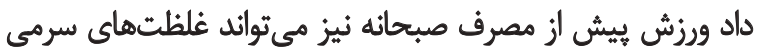

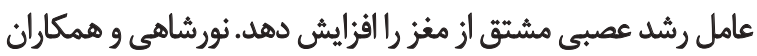

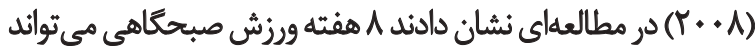

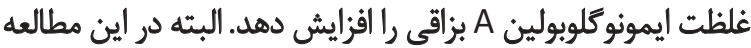

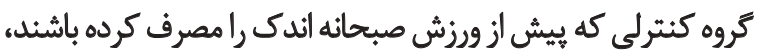

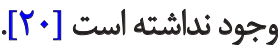

يكى از اهداف افرادى كه در اوايل صبح ورزش مي مكنيند،

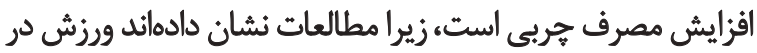

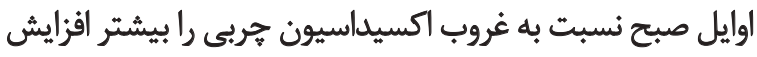

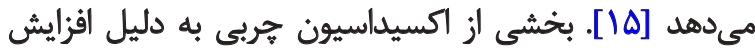

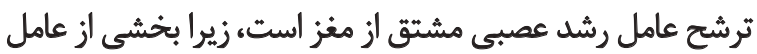

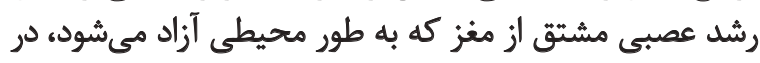

اثر كمخورى "ا يا كاهنده اشتهاى عامل رشد عصبى مشتق از

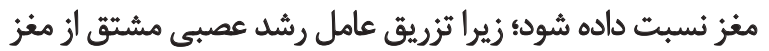

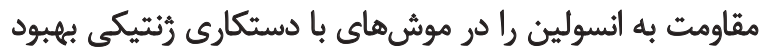

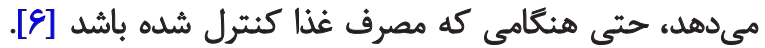

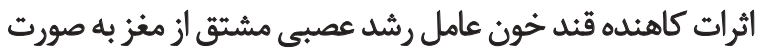

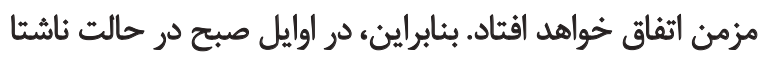

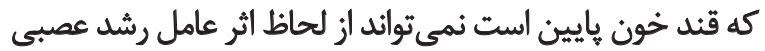

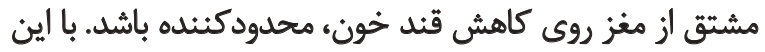

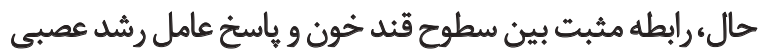

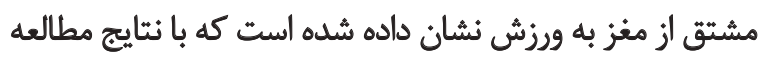

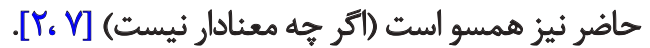
با اينكه افزايش سطوح كلسترول سرمى مى ثواند اثرات مضرى

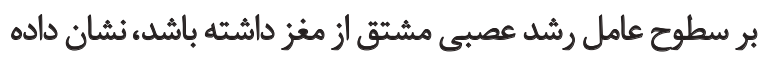

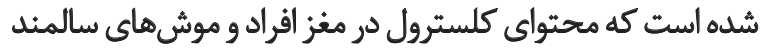

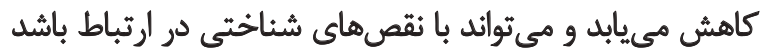

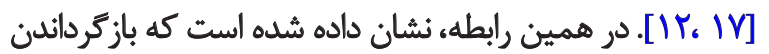

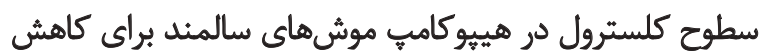

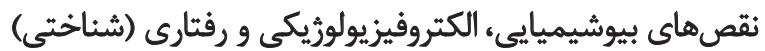

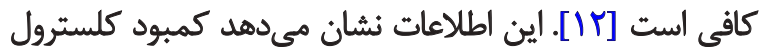

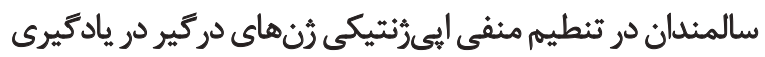

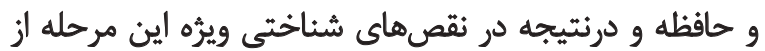

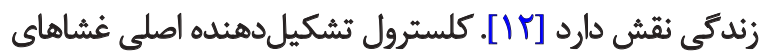

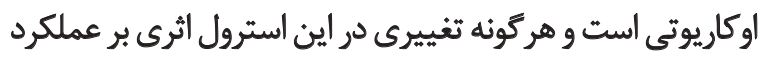

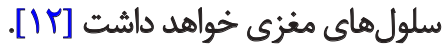

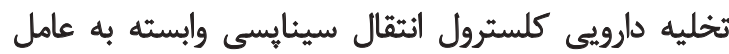

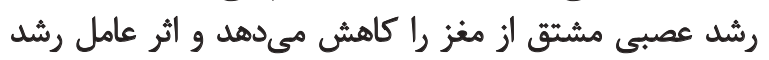

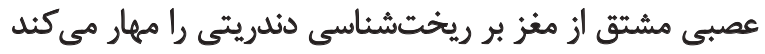

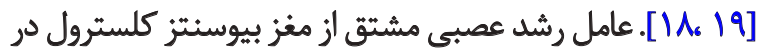

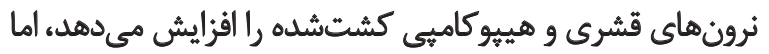

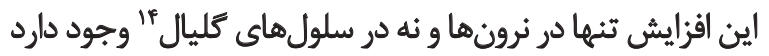

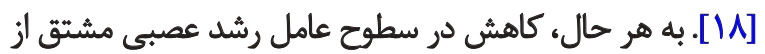

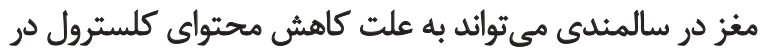

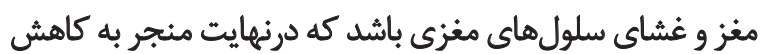
عملكرد شناختي در افراد سالمند مي شئشود.

تضاد در اثر سطوح كلسترول سرمى و كلسترول داخل مغز

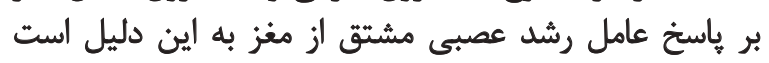

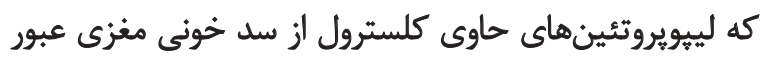

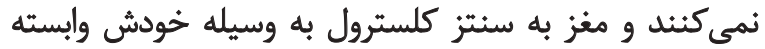

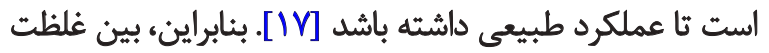

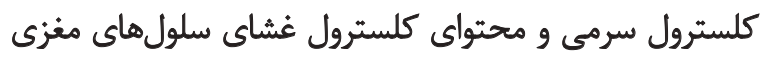

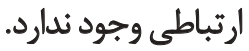

13. Hypophagic

14. Glial 


$$
\text { تشكر و قدودانى }
$$

بدين وسيله از تمامى آزمودنى هاى مطالعه حاضر به خاطر شركت

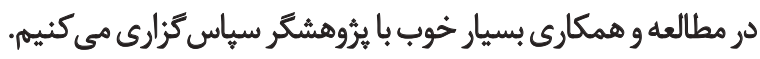

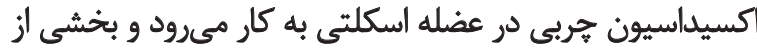

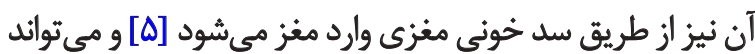
اثرات سودمند ديكرى بر ساختار و عملكرد مغز داشته مانه باشد.

يكى از محدوديتهاى اين مطالعه استفاده نكردن از طرح

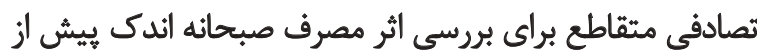

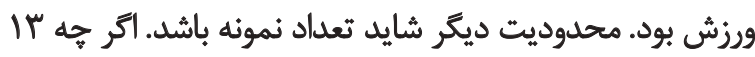

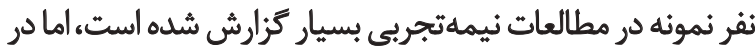

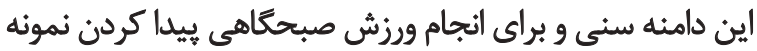

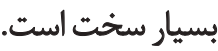

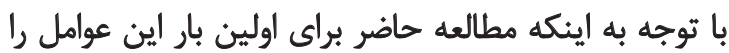

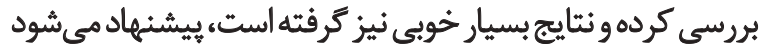

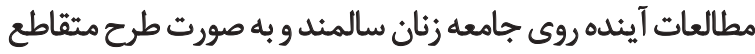

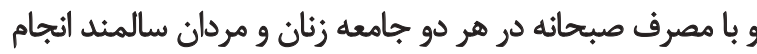

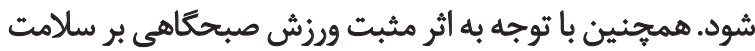

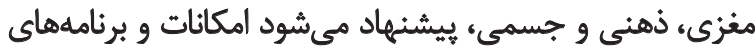

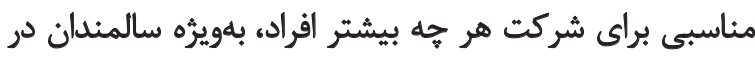

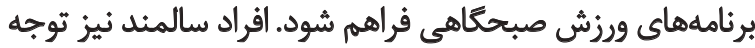

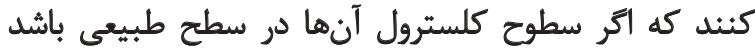

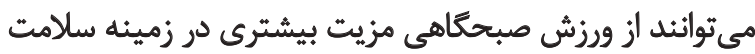

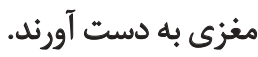

\section{تتيجه كيرى نهايی}

نتايج مطالعه حاضر نشان داد ورزش در اول صبح در حالت

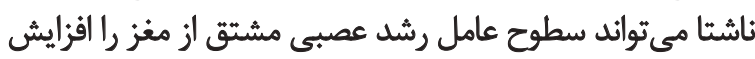

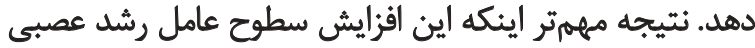

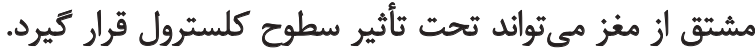

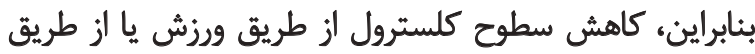

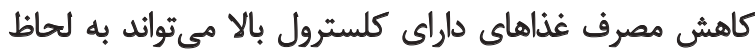
سلامت مغزى و ذهنى اهميت فراوانى داشته باشد.

\section{ملاحظات اخلاقى}

$$
\text { ييروى از اصول اخلاق يثوهش }
$$

كميته اخلاق دانشعاه لرستان اين برؤهش را ثأييد كرد.

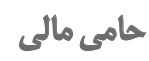

اين بثوهش از طرح درون دانشعاهى دانشكاه لرستان كرفته

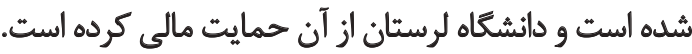

$$
\text { تعارض مثافع }
$$

بنا بر اظهار نويسندكان اين مقاله تعارض منافع ندارد. 


\section{References}

[1] Huang T, Larsen KT, Ried-Larsen M, Møller NC, Andersen LB. The effects of physical activity and exercise on brain-derived neurotrophic factor in healthy humans: A review. Scandinavian Journal of Medicine \& Science in Sports. 2014; 24(1):1-10. [DOI:10.1111/sms.12069] [PMID]

[2] Suzuki T, Shimada H, Makizako H, Doi T, Yoshida D, Ito K, et al. A randomized controlled trial of multicomponent exercise in older adults with mild cognitive impairment. PLoS ONE. 2013; 8(4):e61483. [DOI:10.1371/journal.pone.0061483]

[3] Chang YK, Alderman BL, Chu CH, Wang CC, Song TF, Chen FT. Acute exercise has a general facilitative effect on cognitive function: A combined ERP temporal dynamics and BDNF study. Psychophysiology. 2016; 54(2):289-300. [DOI:10.1111/psyp.12784] [PMID]

[4] Veasey RC, Haskell-Ramsay CF, Kennedy DO, Tiplady B, Stevenson EJ. The effect of breakfast prior to morning exercise on cognitive performance, mood and appetite later in the day in habitually active women. Nutrients. 2015; 7(7):5712-32. [DOI:10.3390/ nu7075250] [PMID] [PMCID]

[5] Coelhoa FGM, Vitala TM, Steina AM, Arantesa FJ, Ruedac AV, Camarinic R, et al. Acute aerobic exercise increases brain-derived neurotrophic factor levels in elderly with Alzheimer's disease. Journal of Alzheimer's Disease. 2014; 39(2):401-8. [DOI:10.3233/ JAD-131073] [PMID]

[6] Karczewska-Kupczewska M, Kowalska I, Nikolajuk A, Adamska A, Zielinska M, Kaminska N, et al. Circulating brain-derived neurotrophic factor concentration is downregulated by intralip$\mathrm{id} /$ heparin infusion or high-fat meal in young healthy male subjects. Diabetes Care. 2012; 35(2):358-62. [DOI:10.2337/dc11-1295] [PMID] [PMCID]

[7] Farooqui T, Farooqui AA. Diet and exercise in cognitive function and neurological diseases. Hoboken, New Jersey: Wiley-Blackwell; 2015. [DOI:10.1002/9781118840634]

[8] Marosi K, Mattson MP. BDNF mediates adaptive brain and body responses to energetic challenges. Trends Endocrinol Metab. 2014; 25(2):89-98. [DOI:10.1016/j.tem.2013.10.006] [PMID] [PMCID]

[9] Vella CA, Kravitz L, Janot JM. A review of the impact of exercise on cholesterol levels. IDEA Health \& Fitness Source. 2001; 19(10):48-54.

[10] Mohammadi MM, Esmaeilivand M. [Attitudes toward caring of the elderly from the perspective of nursing and midwifery students in Kermanshah Province in 2015 (Persian)]. Iranian Journal of Ageing. 2017; 11(4):476-83.

[11] Benloucif S, Orbeta L, Ortiz R, Janssen I, Finkel SI, Bleiberg J, et al. Morning or evening activity improves neuropsychological performance and subjective sleep quality in older adults. Sleep. 2004; 27(8):1542-51. [DOI:10.1093/sleep/27.8.1542] [PMID]

[12] Palomer E, Martin-Segura A, Baliyan S, Ahmed T, Balschun $D$, Venero $C$, et al. Aging triggers a repressive chromatin state at BDNF promoters in hippocampal neurons. Cell Reports. 2016; 16(11):2889-900. [DOI:10.1016/j.celrep.2016.08.028] [PMID]

[13] Perrey S. Promoting motor function by exercising the brain. Brain Sciences. 2013; 3(1):101-22. [DOI:10.3390/brainsci3010101] [PMID] [PMCID]
[14] Yarrowa JF, Whitec LJ, McCoya SC, Borst SE. Training augments resistance exercise induced elevation of circulating Brain Derived Neurotrophic Factor (BDNF). Neuroscience Letters. 2010; 479(2):161-5. [DOI:10.1016/j.neulet.2010.05.058] [PMID]

[15] Robertson T, Palmer R, Doyle A, Griffin B, Hampton S, Collins A. Morning exercise appears to promote greater fat oxidation and reduce postprandial lipaemic response more than evening exercise. Proceedings of the Nutrition Society. 2011. [DOI:10.1017/ S0029665111004794]

[16] Jung SH, Kim J, Davis JM, Blair SN, Cho HC. Association among basal serum BDNF, cardiorespiratory fitness and cardiovascular disease risk factors in untrained healthy Korean men. European Journal of Applied Physiology. 2011; 111(2):303-11. [DOI:10.1007/s00421-010-1658-5] [PMID]

[17] Fukui K, Ferris HA, Kahn CR. Effect of cholesterol reduction on receptor signaling in neurons. Journal of Biological Chemistry. 2015; 290(44):26383-92. [DOI:10.1074/jbc.M115.664367] [PMID] [PMCID]

[18] Suzuki S, Kiyosue K, Hazama S, Ogura A, Kashihara M, Hara $\mathrm{T}$, et al. Brain-derived neurotrophic factor regulates cholesterol metabolism for synapse development. Journal of Neuroscience. 2007; 27(24):6417-27. [DOI:10.1523/JNEUROSCI.0690-07.2007] [PMID]

[19] Gonzalez A, Moya-Alvarado G, Gonzalez-Billaut C, Bronfman FC. Cellular and molecular mechanisms regulating neuronal growth by Brain-Derived Neurotrophic Factor. Cytoskeleton. 2016; 73(10):612-28. [DOI:10.1002/cm.21312] [PMID]

[20] Nourshahi M, Hovanloo F, Arbabi A. [Effect of exercise with moderate intensity in the morning on some factors of immune systems in adults (Persian)]. Iranian Journal of Endocrinology \& Metabolism. 2008; 10(3):241-5. 